\title{
Prediction of Radioactive Injection Dosage for PET Image
}

\author{
Ebtesam Alsanea ${ }^{1}$, Wadee Alhalabi ${ }^{1,2}$ \\ ${ }^{1}$ Department of Computer Science, King Abulaziz University, Jeddah Saudi Arabia. \\ ${ }^{2}$ Department of Computer Science, Effat University, Jeddah Saudi Arabia.
}

\begin{abstract}
An important aspect of PET imaging in clinical application is the localization and detection of tumors and lesions by administering a predetermined amount of radiotracer. This allows for example, a detailed view of what is going inside the patient body in cellular level. The quality of PET image is strongly dependent on the amount of administrated radiotracer and the patient's body parameters. As the amount of injection radiotracer increases, the quality of resulting image increases and the lesion detection efficiency increases. The PET examiner society recognizes that any dose of radiotracer is associated with some possible radiation risks. It can harmful to the patient if essential PET imaging session is not made due to fear of radiation risk. In order to ensure the highest quality diagnosis and the smallest radiation risk, the patient should receive the smallest amount of radiotracer that provide image with sufficient quality.

Our study is focused on proposing an efficient PET simulation tool that predict the smallest possible amount of administrated radiotracer to provides the appropriate diagnostic information based on significant patient's body parameters (weight, age) at fixed scanning to improve the clinical diagnostic process in term of tumor detecting and localization.

We have built a model of particular PET scanner and model of patient based on real MRI image and digital anthropomorphic phantom of our region of interest (brain). We have performed Monte Carlo simulation for whole PET procedure with special parameter In evaluating stage, a dataset of 60 patient is used and 11 independent dose prediction simulation for each patient are performed. We conclude that our simulator perform a desirable and efficient prediction of injection radiotracer amount that optimizes the current clinical amount up to $28 \%$. In addition, we found that the total injected radiotracer dosage for adult patients are mostly affected when considering patient weight rather than patient age.
\end{abstract}

Keywords: Positron emission tomography, Prediction of radioactive injection dosage, PET image, Monte Carlo Simulation.

\section{Introduction}

Medical imaging is becoming an increasingly important component of clinical applications and research oncology for diagnosis treatment planning, and tumor monitoring. During the years, multiple imaging technologies have been developed using the rapid advances in computer and imaging approaches such as : X-ray radiography, Computed Tomography (CT) , Ultra Sound(US) , Magnetic Resonance imaging (MRI), and Nuclear Medicine Imaging : Positron Emission Tomography (PET) and Single Photon Emission Computed Tomography (SPECT)[1], [2].

Nuclear Medicine Imaging is an independent medical imaging field designed to observe the biological function of the body. It is widely applied in the field of cardiology, oncology and neuroscience. This method integrate two main components in order to work properly: radiotracer and detector or scanner.

The radiotracer is a label substance made up of a molecule of interest from the patient's body. It is usually introduced to the body by injection, swallowing or even by annihilation while the scanner is represented by a camera able to detect the radiotracer activity presence and generate images.

PET is described as one of the main applications of Nuclear Medicine Imaging that estimates the spatial distribution of the injected radiotracer based on annihilation of the photons emitted by positron emitting isotopes e.g. C-11, N-13, O-15 and F-18. The information provided by PET differs from other medical imaging modalities such as X-ray, CT, or MRI. These modalities provide anatomical information about the structural changes while PET provides information about biological activities.

Because the biological changes exceed the structural changes, PET let determines the presence of abnormality much earlier than the other approaches[3]-[5].

In PET imaging procedure, a small amount of radiotracer should be injected to the patient in order to examine the body functional process. The PET examiner society recognizes that any dose of radiotracer used this procedure is associated with some possible radiation risks. Radiation dose for PET imaging should be minimized so the patient must receive the smallest amount of radiotracer that provide image with sufficient quality. Suppose that, if essential PET imaging session (PET imaging session that can 
provide to the medic important data about the patient's treatment and diagnosis) is not made due to fear of radiation risk. What will happen? It may harm the patient.

For that reason, also in order to ensure the highest quality diagnosis and the smallest radiation risk, the right PET imaging procedure with the right injection dose should be given to the right patient at the right time.

Because the advantages of PET examination more important than the potential risk. The goal of this paper is to propose a tool to simulate the PET examination session based on significant patient's body parameters (weight, age) at fixed scanning time. This tool predicts the optimal radiotracer injected dosage for individual patient to reduce PET imaging cost and save patients from potential risks.

To achieve our goal, we will describe in the following section the details of PET imaging simulation, the PET scanner model, the patient model as well as explain the PET image quality measurement. Finally, we will predict the smallest possible amount of administrated radiotracer that provides the appropriate diagnostic information to improve the clinical diagnostic process in term of tumor detecting and localization.

\section{PET and Monte Carlo Simulation}

PET imaging deals with radiotracer decay, positron emission, photon transportation and particles detection, the use of simulation is very popular in PET research and clinical practice. Monte Carlo simulation technique plays an essential role in this field and it is covering a wide range of problems that could not solved with experimental or analytical method. Monte Carlo simulations are widely used for PET imaging simulation because they take into account all the random processes concerning PET imaging. PET simulation tools using Monte Carlo are useful in quantifying the radiotracer amount and planning the radiotracer dosage, as well as studying the factors that affect the quality of PET reconstructed image. Furthermore, the Monte Carlo simulation can create data very close to those obtained from real measurement. Currently available simulation tools are not easy to understand and use by beginners and temporary users with no programming and physical background. Also, those software are extremely time consuming, require large space on the hard disk and in general are consumers of high computational resources For example, to simulate a scan of whole body, more than $17000 \mathrm{~h}$ CPU time is required[6], [9].

\section{Injection Dose Prediction}

This section provides an overview of studies relevant to this study .Recently, Boldys et al. employed a Monte Carlo simulation to calculate the amount of injection radiotracer need to achieve statistical constant quality of resulting PET image even with patient with different body parameter (weight). The experiment is done on simple patient model, they used the cylindrical geometric shape to approximate the body trunk, this way is not representative[10], [11].

Also, Karkestani et al. Employed Monte Carlo simulation to model a direct relation between administrated activity and statistical quality measurement (NECR) for a wide range of scanner parameters. They employed digital voxelized phantom and resize it in to (large, small, medium) size phantom. The resulted total amounts of injected radiotracer represented by this work are small than the minimum amount should be administrated to the real patient[12].

\section{PET Simulation Methodology}

This section describes the significant steps to build the proposed PET simulation tool and how it implement the PET physical operations. Figure 1 presents the computerized PET imaging system which is composed of phantom specifications (patient model) and scanner specifications, processed by simulation software in order to reconstruct the PET desired images. Phantoms are seen as a collection of digital volume arrays or 2D images that are used to approximate the locations and dimensions of the patient's body structures and organs. A scanner model designed to illustrate a common PET scanners currently used for clinical examinations. The process of PET imaging is then simulated based on simulation technique such as the Monte Carlo method by following the basic algorithm provided in[13].PET processes like: radiotracer activity uptake, acquisition time, positron range, positron 
inhalation, photon transportation, and photon detection. The information that comes from the simulation is organized in a matrix called sinogram. Sinogram is a simple ordered way to store the events registered by each detector into sets of parallel projections. Finally, using a reconstruction method, the PET image is produced of the injected radiotracer activity distribution within required part of patient's body.

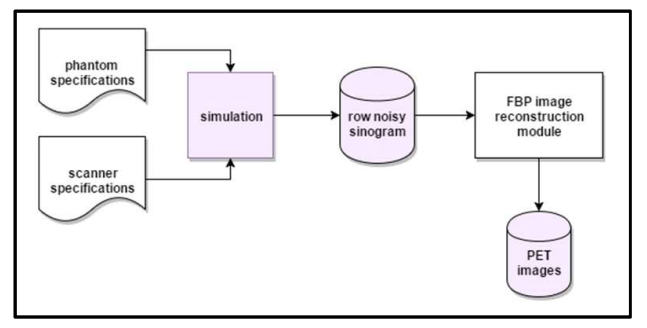

Figure 1. PET simulation tool structure

\section{Patient Model}

Generating a realistic model of the patient's anatomical and biological functions from imaging data is the important aspect of simulation[7], [9], [14] .Theoretically, the patient computerized model represents a tissue, organs and body region in order to visualizing the radiotracer distribution, scattering and absorbing as similar as in real patient. By using a computerized model of patient, we were able test different or completely innovative cases by simply altering that model with different situations. As shown in figure 2, two digital volumes were used for the Monte Carlo simulation performed: a matrix generated from reading a real clinical data [clinical CT or MRI image for the patient] and a matrix of digital anthropomorphic phantom [mathematical model design to accurately represent the tissue and organs system in the whole body]. Clinical data provides the approximate accurate experimental results, while digital phantom provides the simulation data for evaluation of detecting abnormal lesions and tumors.

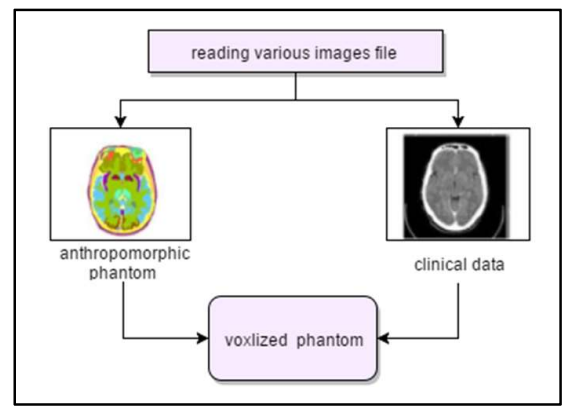

Figure 2. Digital voxlized phantom

\section{Radiotracer Injection Dose Calculation}

The radiotracer used in PET imaging can affect the quality of resulting images. All radiotracers used in PET have to satisfy some requirements such as: they have to be not toxic or harmful for the patient, they have to be chemically incorporated into the biological process under examination without modifying it. In addition, they should to be specific for the physiological process under study. The most common radiotracer used in PET examination is Floor-Deoxy-Glucose (FDG). As FDG is analogous to glucose and tumours accumulate glucose more than the surrounded healthy tissues, it is used to stage cancer and to detect possible tumours[3], [5].

The amount of radiotracer that should to be injected to a patient's body is an important issue in PET examination. There are standards on what the dosage amount of radiotracer that must be administrated. In this regard and according to the European Association of Nuclear Medicine EANM , the amount of injected FDG radiotracer applied in the scan for adult patient approximately calculated by equation (1) [15] 


$$
I A=7.2 * \text { patient weight }(K G) / \text { scan time }(\min ) \pm 10
$$

By flowing the principle of as low as reasonable achievable (ALARA) [16]each patient should receive the minimum amount of IA that is necessary to produce a good diagnostic image. To this purpose and along with the variation in patient body parameters, we proposed a method to scale the IA dose according to patient's age based on EANM guideline recommendation and Young's formula used in nuclear medicine dosage calculation[17]-[19]:

$$
I^{\prime}=\left[\frac{\text { patient age }(\text { year })}{\text { patient age }(\text { year })+12}\right] * \text { IA }
$$

\section{Scanner Model}

When defining the model of a tomographic scanner, specific guidelines with respect to the hierarchy of the scanner component must be followed in order to track the particle's physical interactions. Most PET scanners are built from single or multiple rings, each ring having several blocks divided into crystal detectors. The number of crystal detectors in blocks for single ring computing as following:

$$
\text { No. Detectors }=\frac{2 \pi * \text { RingRadius }}{\text { DetectorSurfaceArea }}
$$

According to[20], the ring radius is usually setting between 300 and $600 \mathrm{~mm}$ and the surface area of each crystal detector is usually setting between 2 and $5 \mathrm{~mm}$.

After setting up the scanner model, we define the PET physical processes that occur during the simulation. The four processes we implementing are summarize in Figure 3 and the detailed description of each process can be found in[21].

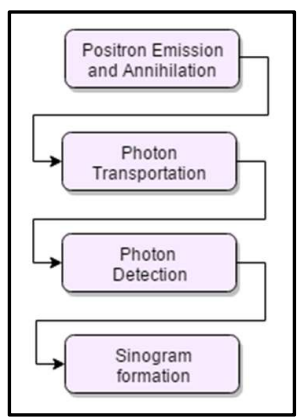

Figure 3.PET physical processes implementing in the simulation

\section{Image Quality Measurement Tools}

There are many image quality assessments tools available in the field of PET reconstructed image expressing and evaluation. For the purpose of our work, we are choosing to evaluate the two most popular statistical tools: Signal to Noise Ratio (SNR) and Noise Equivalent Count (NEC). from medical point of view, for a given diagnostic task such as tumor/lesion detection, the significant measure for PET image quality is observing and detecting small foci[10], [11], we provide a particularly attractive performance measurement of the reconstructed image precision and recall.

SNR is a statistical measurement representing the relation between an acquired signal and the background image noise. We are getting its value by calculating the ratio of total detected photons mean to the signal standard deviation.

$$
S N R=20 \log \left(\frac{D P \mu}{D P \sigma}\right)
$$

NEC is a statistical measurement describing the number of detected photons needed to produce an equivalent image with PET ideal system .It is obtained by calculating the ratio between the numbers of detected photon to the total number of photon events. 


$$
N E C=\left(20 \log \left(\frac{D P \mu}{D P \sigma}\right)\right)^{2}
$$

The ultimate measure of PET image quality is the ability to detect the presence of possible tumors and lesions. In a certain situation The diagnostic task of classifying significant changes of the pixel values of the reconstructed image according to the given region can be treated as a problem of tumor detection and location. There are several measures of performance for the tumor detecting classifiers such as precision and recall. In order to calculate precision and recall, the classifier confusion matrix is computed. As shown in Figure 4.

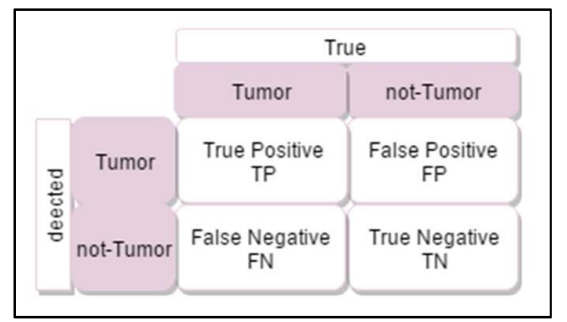

Figure 4. Confusion matrix

Precision measures the percentage of the pixels detected as tumors that are truly tumors. Recall measures the percentage of tumor-pixels that are truly detected from all pixels in the reconstructed image. The following equations represent the precision and recall, computed based on the confusion matrix:

$$
\begin{gathered}
\text { precision }=\frac{\boldsymbol{T P}}{\boldsymbol{T P}+\boldsymbol{F P}} \\
\text { recall }=\frac{T P}{T P+F N}
\end{gathered}
$$

\section{PET simulation Experiments}

In this section, different simulations on a set of patients chosen from clinical data are performed. We have 60 patients MRI images and metadata from available open source Digital Imaging and Communication in Medicine DICOM library. The main purpose of these simulations is to evaluate the simulated PET system discussed in previous sections and then predict the optimal radiotracer injection dosage used in detecting abnormal lesions and tumors.

To perform our simulations, the patient model is built from digital phantom and his clinical MRI image. By following the algorithm illustrated in Figure 5, each patient model is injected with 11 independent different 18F-FDG dosages calculated from equation (2). The scanner model is also built according to the technical specifications of real clinical scanner used for PET imaging system. After that, the PET imaging simulation is done for all patient models. Furthermore, according to the experiment scope, the patient models are divided into three different groups based on their age and weight. Finally, the resulting images and statistical values for those groups are used in system evaluation comparisons, image quality assessments and performance measurements. .

In this simulation, we choose brain as our main region of interest because it important region for PET imaging in clinical examination. In addition, the brain has frequent lesions and tumor occurrence beside other abnormality disease including Parkinson's disease and Alzheimer's disease. 


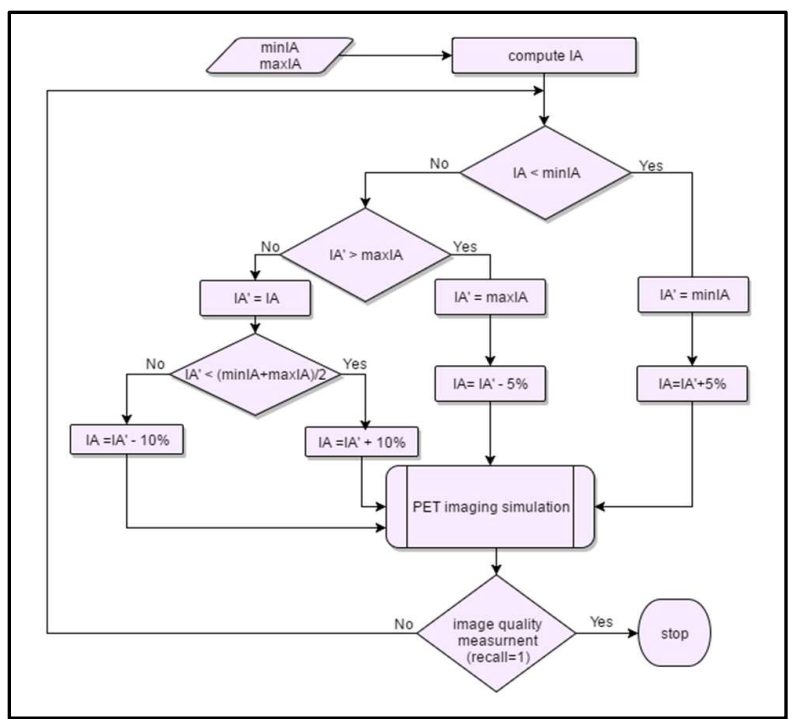

Figure 5.Radiotracer injection dose calculation process

\section{PET simulation Results and Discussions}

We simulated a set of 60 patients provided in the DICOM data set with the range of weight from 40 to $150 \mathrm{Kg}$, and adult age's distribution from 20 to 95 . From the given weight range and for constant age, we can divide the patients dataset into four groups: slim patients (group of patients with very low weight, $45 \geq$ weight), medium-weight patients (group of patients with moderate weight, $45<$ weight $<70$ ), obese patients (group of patient with a heavy weight, $70<$ weight $<90$ ) and overweight patients (group of patient with weight $<90$ ). The PET image simulations are classified as following: 1) Computing the NEC for default patient parameters weight $=63 \mathrm{~kg}$ and age 35 years (Figure 6). 2) Calculating the optimal radiotracer injection amount for patient parameters weight $=63 \mathrm{~kg}$ and age 35 years (Figure 7). 3) Calculating the optimal radiotracer injection amount for patients with different weight in $\mathrm{Kg}$, and constant age in year ((Figure 8) .4) Calculating the optimal radiotracer injection amount for patients with fix weight in $\mathrm{Kg}$ and different ages in year (Figure 9).

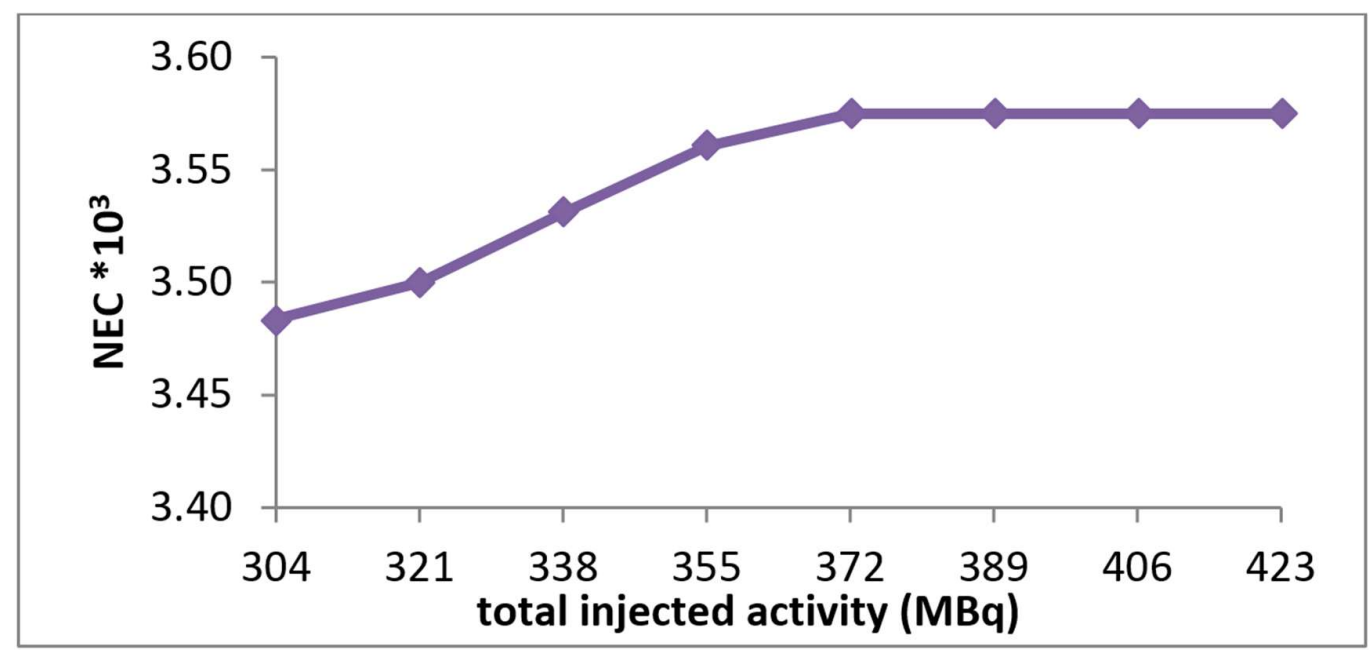

Figure 6. NEC values for default scanner and patient model 


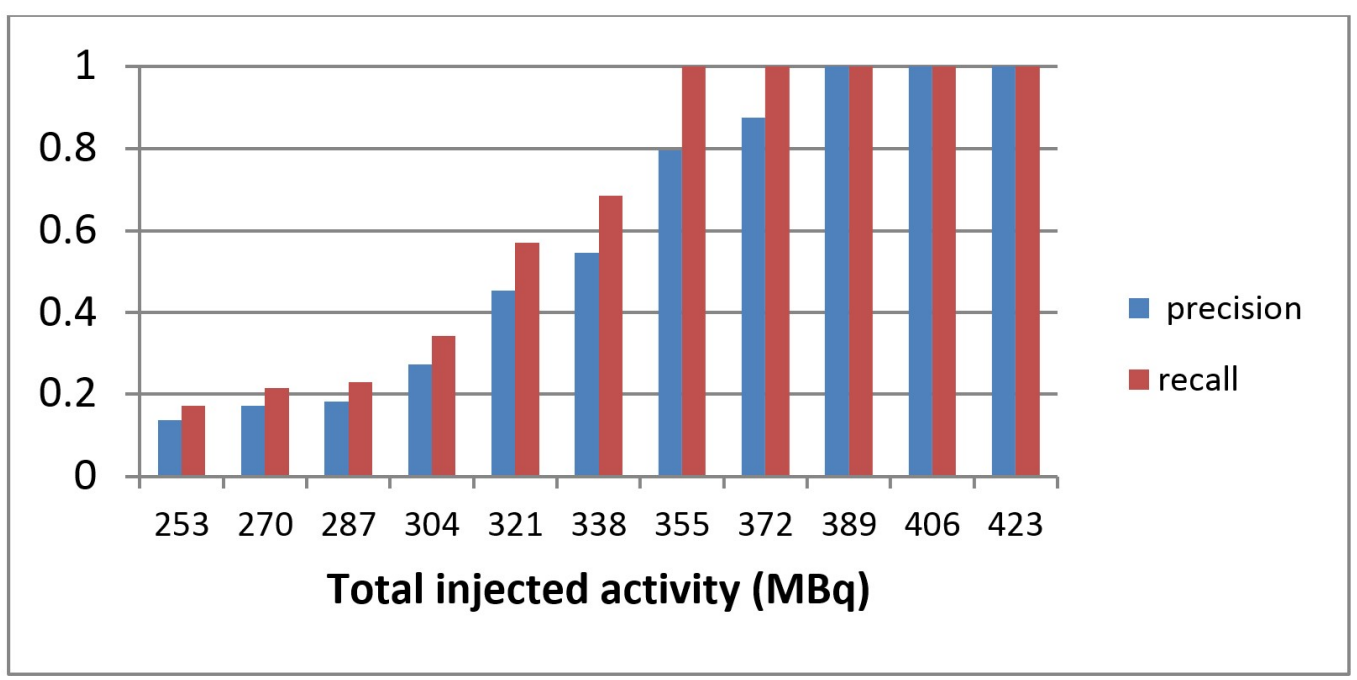

Figure 7. Lesion detection (precision and recall) for default patient model

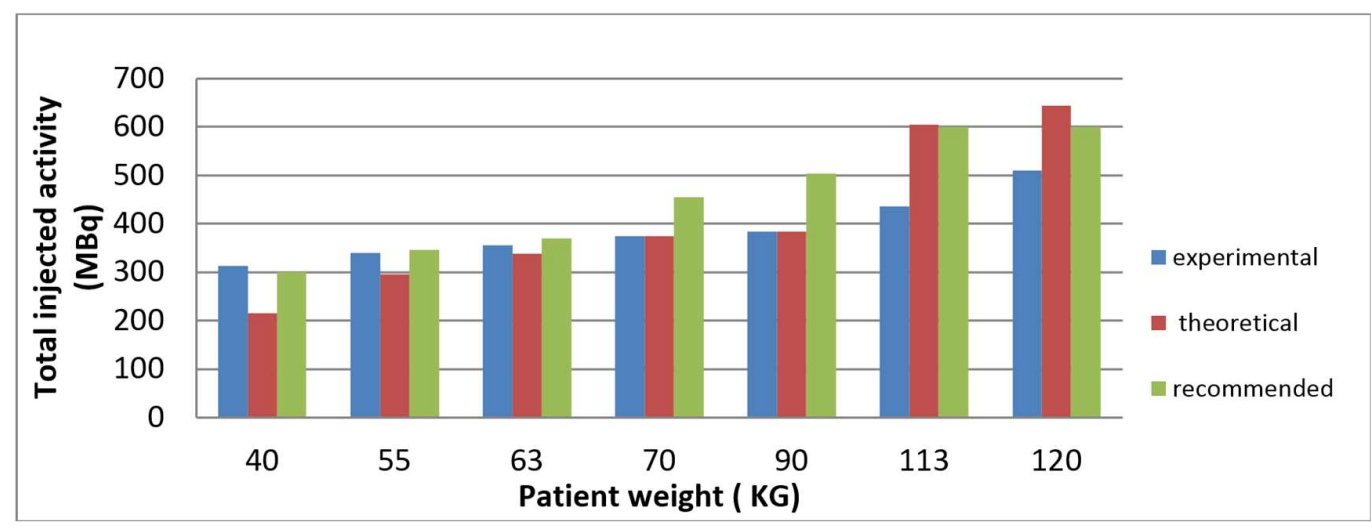

Figure 8.Total injected activity for patients with different weight $(\mathrm{Kg})$ and fixed age ( $=35$ years)

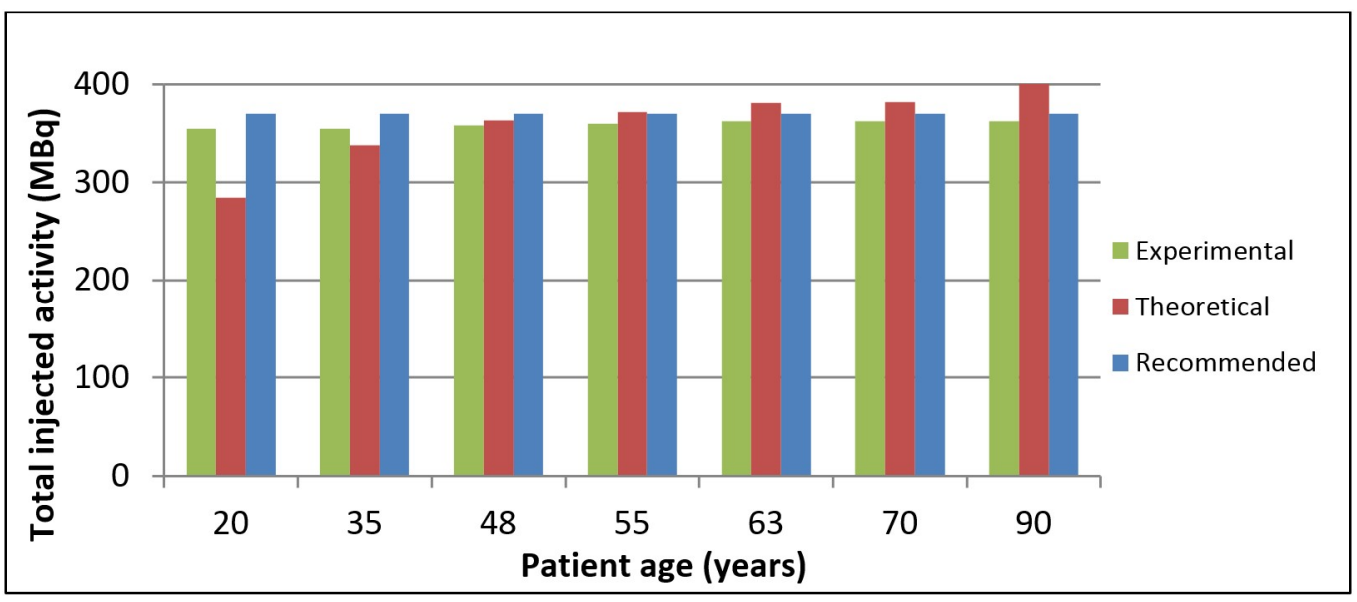

Figure 9.Total injected activity for patients with fixed weight $(\mathrm{Kg})$ and different age (years) weight $=63 \mathrm{Kg}$ 
Figure 6 as the injected amount of radiotracer increase the quality of the reconstructed PET image is increase. After the peak of NEC values is reached then as the injected amount of radiotracer increase the NEC values are remaining the same and the quality of the reconstructed PET image will remaining also. For the same patient, Figure 7 illustrates the evaluation results of calculating the optimal radiotracer injection amount in term of lesion and tumor detection based on precision and recall values. The optimal radiotracer amount according to our simulation is $355 \mathrm{MBq}$ that is $5 \%$ optimized compared to the clinical injection dosage which equals to $370 \mathrm{MBq}$.Also, it discrepancy from the calculated initial dosage value 338 Mobs by $5 \%$.

Figure 8 illustrates the evaluation results of calculating the optimal radiotracer injection amount in terms of lesion and tumor detection based on precision and recall values for a set of randomly selected patients from the available dataset of 60 patient's .The comparisons of the simulator, recommended and theoretical calculating injected dosage according to the patients groups as following:

- For the group of slim patients, the minimal injected radiotracer amount by the simulator is $4 \%$ greater than recommended dose

- For the group of medium weight patients, the simulator dosage are optimizing the clinical recommendation approximately by $5 \%$ to $7 \%$

- For the group of obese patients, the simulator optimizing the clinical recommendation dosage by $17 \%$ to $24 \%$.

- For the group of overweight patients, the simulator dosage are optimizing the clinical recommendation dose up to $28 \%$.

Finally, Figure 9 illustrates the evaluation results of calculating the optimal radiotracer injection amount in terms of lesion and tumor detection based on precision and recall values for a set of randomly selected patients. The comparative evaluation between simulator, clinical recommendation and the theoretically calculated dosage shows a discrepancy values for the theoretical dosage among patients. Also, the simulator dosage provides $4 \%$ to $5 \%$ optimization to the clinically recommended dosage of injected radioactive substance.

\section{Conclusion}

The main purpose of this study is to predict the optimal radiotracer injected dosage for adult patients. To achieve our goal, we performed a set of simulations to assessing the relationship between the quality of simulated PET image, the amount of radiotracer injected to the patient and the patient's physical parameters (age, weight). Each simulation is done using the PET scanner model and the patient model to diagnostic task (tumor/lesion detection), it calculates the PET image quality assessment variables NEC and SNR. From the results of those simulations, we conclude that our simulator can drive a desirable and efficient prediction of injection radiotracer amount that optimizes the current clinical amount up to $28 \%$. In addition, we can conclude that the total injected radiotracer dosage for adult patients are mostly affected by the patient weight rather than by the patient age.

\section{References}

[1] S. R. Cherry, "Multimodality imaging: Beyond pet/ct and spect/ct," in Seminars in nuclear medicine, 2009, vol. 39, pp. 348-353.

[2] O. Mawlawi and D. W. Townsend, "Multimodality imaging: an update on PET/CT technology," Eur. J. Nucl. Med. Mol. Imaging, vol. 36, no. 1, pp. 15-29, 2009

[3] S. E. de O. B. Silva, "Small animal PET imaging using GATE Monte Carlo simulations: Implementation of physiological and metabolic information," 2010

[4] J. E. Gillam and M. Rafecas, "Monte-Carlo simulations and image reconstruction for novel imaging scenarios in emission tomography," Nucl. Instrum. Methods Phys. Res. Sect. Accel. Spectrometers Detect. Assoc. Equip., vol. 809, pp. 76-88, 2016.

[5] A. Turco, "Monte Carlo simulations of a small-animal PET scanner. Analysis of performances and comparison between camera designs," 2012.

[6] H. Zaidi, "Relevance of accurate Monte Carlo modeling in nuclear medical imaging," Med. Phys., vol. 26, no. 4, pp. 574-608, 1999.

[7] M. Ljungberg, "Simulation Techniques and Phantoms," in Emission Tomography, The Fundamentals of PET and SPECT, Elsevier Inc., 2004, pp. 551-563.

[8] H. Zaidi and G. Sgouros, Therapeutic applications of Monte Carlo calculations in nuclear medicine. CRC Press, 2002.

[9] H. Zaidi and K. Koral, "Scatter Correction Strategies in Emission Tomography," in Quantitative Analysis in Nuclear Medicine Imaging, Springer Science, 2006, pp. $205-235$.

[10] J. Boldyš, J. Dvořák, M. Skopalová, and O. Bělohlávek, "Monte Carlo simulation of PET images for injection dose optimization,” Int. J. Numer. Methods Biomed. Eng., vol. 29, no. 9, pp. 988-999, 2013.

[11] J. Dvořák, J. Boldyš, M. Skopalová, and O. Bělohlávek, "Application of the random field theory in PET imaging-injection dose optimization," Kybernetika, vol. 49, no. 2, pp. 280-300, 2013. 
[12] N. A. Karakatsanis, G. Loudos, A. Rahmim, and K. S. Nikita, "Monte-Carlo based characterization of the counting rate (NECR) response for personalized optimization of the administered activity in clinical PET imaging," Front. Biomed. Technol., vol. 1, no. 1, pp. 14-34, 2014

[13] S. Yu, "Simulation of PET Brain Images Using Monte Carlo Method," Master Thesis in Medical Imaging, School of Technology and Health Royal Institute of Technology, Stockholm, Sweden, 2010

[14] J. Peter, M. Tornia, and R. Jaszczek, "Analytical versus voxelized phantom representation for Monte Carlo simulation in radiological imaging.," Medical Imaging, IEEE Transactions on, vol. 19, no. 5, pp. $556-564,2000$.

[15] R. Boellaard, M. J. O'Doherty, W. A. Weber, F. M. Mottaghy, M. N. Lonsdale, S. G. Stroobants, W. J. Oyen, J. Kotzerke, O. S. Hoekstra, and J. Pruim, "FDG PET and PET/CT: EANM procedure guidelines for tumour PET imaging: version 1.0," Eur. J. Nucl. Med. Mol. Imaging, vol. 37, no. 1, pp. 181-200, 2010.

[16] K. M. Waterstram-Rich and P. E. Christian, Nuclear medicine and PET/CT: technology and techniques. Elsevier Health Sciences, 2013.

[17] A. Piepsz, K. Hahn, I. Roca, G. Ciofetta, G. Toth, I. Gordon, J. Kolinska, and J. Gwidlet, "A radiopharmaceuticals schedule for imaging in paediatrics," Eur. J. Nucl. Med., vol. 17, no. 3-4, pp. 127-129, 1990.

[18] S. T. Treves, A. Baker, F. H. Fahey, X. Cao, R. T. Davis, L. A. Drubach, F. D. Grant, and K. Zukotynski, "Nuclear medicine in the first year of life," J. Nucl. Med., vol. 52, no. 6, pp. 905-925, 2011.

[19] R. Accorsi, J. S. Karp, and S. Surti, "Improved dose regimen in pediatric PET," J. Nucl. Med., vol. 51, no. 2, pp. 293-300, 2010.

[20] G. B. Saha, Basics of PET imaging: physics, chemistry, and regulations. Springer Science \& Business Media, 2010

[21] Ebtesam Alsanea, "Prediction of Radioactive Injection Dosage for PET Image," king AbdulAziz university, 2016. 